\title{
A Prospective Study on Clinically Significant Drug - Drug Interactions by Systematic Review of Case files in a South Indian Tertiary Care Hospital
}

\author{
Dilip Chandrasekhar, Anju Shivasankaran Nair, Anu Pauly Rose Mathew, Anu Baby, Athira Manikandan \\ Department of Pharmacy Practice, Al Shifa College of Pharmacy, Perinthalmanna, Kerala, INDIA.
}

\begin{abstract}
Objectives: The main objective of the study was to recognize potential drug-drug interactions, thus reducing their potential risk and improving pharmaceutical care by minimizing problems that may arise out of improper medication. Methodology: A prospective interventional study of a duration of 6 months was conducted in three phases (Pre interventional, Interventional and Post interventional) among 200 inpatients (in the age group of 18 years and above) of Pulmonology, Nephrology, Gastroenterology and General Medicine Departments of a tertiary care referral hospital. Results: Out of a total of 495 interactions, $368(74.34 \%)$ interactions occurred in the Pre interventional phase and 127 (25.65\%) interactions in Post interventional phase. There was a statistically significant reduction in drug-drug interactions occurring in the Post interventional phase ( $P$ value $<0.05$ ) which suggests encouraging the intervention of clinical pharmacists in assessing and controlling potential drug-drug interactions. Conclusion: Among the conclusions of this study are that a wide number of DDIs exist within a hospital environment. Further, that a majority of the DDIs may be reduced by a significant amount via means of contact programs with both
\end{abstract}

physicians as well as subjects. This may include distributing information letters to physicians concerned and providing bedside patient counseling as was carried out in this study. In conclusion, our study suggests that a clinical pharmacist could make statistically provable improvements in minimizing harmful outcomes of DDIs by means of calculated study and information disbursal of the given medical scenario.

Key words: Drug drug interactions, Drug Interaction probability scale, Lexicomp, Pharmacodynamic , Pharmacokinetic.

Correspondence

Dr. Dilip Chandrasekhar,

Department of Pharmacy Practice, Alshifa College of Pharmacy, Poonthavanam P.0, Perinthalmanna, Kerala-67932, INDIA.

Phone: +91- 9447252670

Email: dillu7@gmail.com

DOI: 10.5530/jyp.2019.11.57

\section{INTRODUCTION}

Drugs are substances developed for human betterment but at the same time they are proved to cause harm to human life. ${ }^{1}$ Drug-drug interaction is defined as the pharmacokinetic or pharmacodynamic influence of drugs on each other which may either result in desired effects, reduced efficacy and effectiveness or increased toxicity. ${ }^{2}$ Drug-drug interactions are increasingly acquiesced as an area of major concern. Drug-drug interactions are associated with significant morbidity, mortality, impaired quality of life and are primary drivers of hospital admissions. ${ }^{3}$ It remains as a major contributor of health damage within hospitals as too many medications may be prescribed simultaneously particularly in cases of Geriatric patients due to the presence of co-morbidities. Life expectancy has increased tremendously due to the abundance of medicines. Most geriatric patients as well as patients with ancillary conditions require poly pharmacy and hence are at higher risk of drug-drug interactions. ${ }^{4}$ Physicians are not averse to the use of multiple medications to manage complicated diseases. This could result in the proverbial double-trouble of drug-drug interactions which are side effects and difficulties in compliance. It comes as no surprise that Drug-Drug interactions are of a major concern for patients these days. Medication errors because of their severe consequences especially in the long term, has been increasingly gaining attention. Therefore the prevention of its occurrence has become a major priority in health care systems. ${ }^{5}$ This study is anticipated to raise cognizance of preventing drug-drug interactions by assessing clinically significant drug-drug interactions by a systematic review of case files in South Indian tertiary care hospitals. ${ }^{6}$ Assessing and controlling the occurrence of clinically important drug-drug interactions can reduce medication related problems and thereby improve pharmaceutical care. Incidence of drug-drug interactions is estimated to vary from 6\%-30\% among hospitalized patients and still offers a significant risk to patient health status and continues to pose a considerable economic burden on health care system. ${ }^{8}$ Approximately $37-60 \%$ of patients admitted to the hospital may have one or more potentially interacting drug combinations at admission. The HARM study recently reported that $2.4 \%$ of all hospital admissions and 5.6\% of all emergency admissions in Netherlands were related to drug-drug interactions of which almost half were considered preventable. ${ }^{9}$ Factors that have shown consistent association with the presence of potential drug-drug interactions in previous studies included pharmacotherapy, age, gender, main diagnosis, medications and the number of physicians that the patient visits. ${ }^{10}$ Drug-drug interaction is a modification of the effect of a drug when administered with another drug. This effect can be severe, moderate or a mild type and there is also a chance for that effect is not associated with either drug. ${ }^{11}$ The outcome can be harmful if the interaction cause an increase in the toxicity of the drug. For example there is a considerable increase in risk of severe muscle damage if patients on statins start taking azole antifungals. A reduction in efficacy due to an interaction can sometimes be just as harmful as an increase: patients taking warfarin who are given rifampicin need more warfarin to maintain adequate and protective anticoagulation. ${ }^{12}$ DDI's can be classified according to the mechanism by which drugs interact with each other as pharmacokinetic and pharmacodynamic. The drug-drug interactions are assessed and classified based on their severity with the help of interaction checking tools such as Up

This is an open access article distributed under the terms of the Creative Commons Attribution-NonCommercial-ShareAlike 4.0 License, which allows others to remix, tweak, and build upon the work non-commercially, as long as the author is credited and the new creations are licensed under the identical terms. 
to Date, Lexicomp and Stokley's interaction checker. Having knowledge of the most commonly occurring preventable drug-drug interactions, the prevalence and risk factors of clinically important potential drugdrug interactions will help clinicians and other health care professionals to identify patients who are at a higher risk of drug-drug interactions arising out of adverse drug events requiring more cautious pharmacotherapy management to reign in the same. Furthermore health care professionals should be aware of the associated risks and should consciously avoid prescribing unnecessary medications to prevent poly pharmacy from occurring. Poly pharmacy is one of the most avoidable practices that lead to the occurrence of drug-drug interactions. Further physicians should prescribe medicines based on evidence with rational combinations and the pharmacist should accordingly take specialized care so that drug-drug interactions are avoided. This study focuses on creating awareness for preventing drug-drug interactions by assessing clinically significant drug-drug interactions by systematic review of case files in a south Indian tertiary care hospital and is expected to elevate the importance of drug monitoring and review of medication charts of hospitalized patients and thereby help both clinicians and researchers to have better clarity on this matter and perhaps guide clinicians on how to avoid such interactions. It is therefore necessary for healthcare professionals to routinely evaluate the patient medication chart to prevent drug-drug interactions and to simultaneously report it to the physician so that he/ she may take measures to prevent its adverse effects on the health of concerned patients. It has evidently become a challenge for physicians to find time for recognizing drug-drug interactions. Recognizing clinically significant drug-drug interactions can result in improved quality of life and help in improving prescription patterns.

\section{MATERIALS AND METHODS}

\section{Methodology}

A prospective interventional study was carried out in a 750 bedded super specialty tertiary level referral hospital situated in Perinthalmanna, part of Malappuram district of Kerala for a period of six months. The study was conducted among 200 inpatients in the age group of 18 years and above from General Medicine, Pulmonology, Nephrology and Gastroenterology departments. Written informed consent was obtained from the participants prior to the study. Investigators respected and prioritized the privacy and confidentiality of all study participants leaving no chance to disclose their identity outside. Patients hospitalized for more than 24 hrs with two or more drugs in their daily prescriptions were included in the study. Pregnant women, psychiatric patients and patients with a history of drug abuse were excluded from the study. A total of 100 patients each were evaluated in the pre interventional and post interventional phase. An observational audit was conducted during the pre-interventional phase for all patients who were found eligible according to the inclusion criteria for a period of 3 months. All the relevant information was collected from patient case files, medication chart, interviews with patients and care givers. The data were analyzed, evaluated, categorized and recorded in a documentation format.

During the interventional phase, based on the results of data analysis from pre interventional phase, an information letter with a list of commonly occurring DDIs with its significance and severity was prepared and distributed to the physicians of the departments involved in the study. Recommendations for altering therapies and monitoring the laboratory values, vitals etc were suggested to the physicians during this phase of 15 days. This phase was followed by a post interventional phase which extended for a period of 2 and half months, during which the procedures of pre interventional phase were repeated with another randomly selected 100 inpatients from the departments concerned. The results of pre interventional phase and post interventional phase were compared and the impact of the clinical pharmacist's intervention in reducing the drug-drug interactions was analyzed. Data was entered into Microsoft Excel and the recorded data were statistically analyzed using Statistical Package for Social Sciences (SPSS) software version 20.0 for WINDOWS. The collected data from subjects were analyzed by statistical treatment using appropriate statistical tools.

\section{RESULTS}

200 patients were randomly selected from the respective departments and followed during the period of 6 months. A total of 495 drug-drug interactions were found during the study period of which 368 interactions occurred in the pre interventional phase and 127 interactions occurred in the post interventional phase. Out of the total study population, 110 (55\%) were males and 90 (45\%) were females (Figure 1). Male patients were predominant over females in the overall population and also in the study population with drug-drug interactions. From the total study population, $40.5 \%$ were found to be above 60 years and $7 \%$ below 20 years. The mean age was $51.83 \pm 20.86$ years.

The results also showed that majority of the patients with Drug-Drug Interactions (DDIs) were prescribed with 5-9 drugs per prescription (61\%). As per the results from pre interventional and post interventional phase, the number of DDIs increased with age. Majority of drug- drug interactions were seen in patients above 60 years $(40.5 \%)$ and least number of DDIs was found in patients below 20 years (7\%). (Figure 2) The data obtained showed a $P$ value of 0.075 which indicated that the relation between Age and DDIs was statistically significant. There was no significant relationship between gender and drug-drug interactions as the $P$ value obtained was greater than 0.05 . The no. DDIs increased with an increase in number of drugs per prescription. The relationship was highly statistically significant as the obtained $P$ value was less than 0.01 .

Out of 368 DDIs in Phase 1 and 127 DDIs in Phase III, occurrence of major DDIs were $136(74.3 \%)$ and 21 (25.65\%) respectively. Moderate DDIs were 217 (43.83\%) and 98 (19.79\%) and minor DDIs were found to be $15(4 \%)$ and $8(6.29 \%)$ respectively.

Out of the 495 DDIs that occurred in study population of 200 patients, $66(13.33 \%)$ were of X severity, 106 (21.41\%) were of D severity and 274 (55.35\%) were of C severity according to LEXICOMP Risk Rating. The study indicated a reduction in the number of Probable, Possible and Doubtful DDIs after the interventional phase with a $P$ value less than 0.05 which indicated that the data is statistically significant. Majority of DDIs that occurred in the study population was of Pharmacokinetic type (45.45\%) followed by Pharmacodynamic and unknown DDIs. A total of 167 changes in laboratory values were found in the study population of which 118 (70.65\%) was seen in Phase I and 49 (29.34\%) was seen in Phase III. Out of a total of 85 interventions done for DDIs, 69 (81.17\%) was done for interactions that occurred in Phase 1 and 16 (18.82\%) for DDIs that occurred in Phase III.

\section{DISCUSSION}

Assuring patient's safety is a major challenge and opportunity for health care providers as it is a major component in providing quality care and superior healthcare delivery. Clinical Pharmacists have an important role in preventing adverse events from occurring to patients in a clinical setting. Among the adverse events that can occur, DDIs are of major concern.

DDIs can be found among the inpatients that are mostly prescribed with more than two drugs. In Harvard Medical Practice Study of adverse events, $20 \%$ of events in an acute hospital in-patient setting were drug related. Of this $8 \%$ were considered to be due to DDIs. ${ }^{13} \mathrm{~A}$ few of the factors that are found responsible for causing DDIs in a hospital setting 
Table 1: Relationship between number of drugs prescribed and DDIs.

\begin{tabular}{|c|c|c|c|c|c|c|c|c|c|c|}
\hline & \multirow[t]{2}{*}{ PHASE } & & \multicolumn{5}{|c|}{ No. of DDIs/prescription } & \multirow[t]{2}{*}{$\begin{array}{l}\text { Chi } \\
\text { square }\end{array}$} & \multirow{2}{*}{$\begin{array}{c}\text { Df } \\
8 \\
\end{array}$} & \multirow{2}{*}{$\begin{array}{c}P \\
\text { Value } \\
0\end{array}$} \\
\hline & & & No DDIs & $1-2$ & $3-4$ & $5-6$ & Above 6 & & & \\
\hline \multirow{8}{*}{ PHASE 1} & & $<5$ DRUGS & 6 & 3 & 0 & 0 & 0 & \multirow{7}{*}{37.18} & & \\
\hline & & & $42.9 \%$ & $13.6 \%$ & $0.0 \%$ & $0.0 \%$ & $0.0 \%$ & & & \\
\hline & No. of & \multirow{2}{*}{5 -9 DRUGS } & 8 & 16 & 19 & 8 & 11 & & & \\
\hline & Prescription & & $57.1 \%$ & $72.7 \%$ & $70.4 \%$ & $44.4 \%$ & $57.9 \%$ & & 8 & 0 \\
\hline & & \multirow{2}{*}{$>10$ DRUGS } & 0 & 3 & 8 & 10 & 8 & & & \\
\hline & & & $0.0 \%$ & $13.6 \%$ & $29.6 \%$ & $5.5 \%$ & $4.21 \%$ & & & \\
\hline & Total & & 14 & 22 & 27 & 18 & 19 & & & \\
\hline & $100.0 \%$ & & $100.0 \%$ & $100.0 \%$ & $100.0 \%$ & $100.0 \%$ & $100.0 \%$ & \multirow{9}{*}{46.23} & & \\
\hline \multirow{8}{*}{ PHASE 3} & & \multirow{2}{*}{$<5$ DRUGS } & 28 & 2 & 0 & 0 & 0 & & & \\
\hline & & & $58.3 \%$ & $5.6 \%$ & $0.0 \%$ & $0.0 \%$ & $0.0 \%$ & & & \\
\hline & No. of & \multirow[b]{2}{*}{5 -9 DRUGS } & 19 & 30 & 6 & 3 & 2 & & & \\
\hline & $\begin{array}{l}\text { Drugs/ } \\
\text { Prescription }\end{array}$ & & $39.6 \%$ & $83.3 \%$ & $85.7 \%$ & $60.0 \%$ & $50.0 \%$ & & & \\
\hline & & \multirow{2}{*}{$>10$ DRUGS } & 1 & 4 & 1 & 2 & 2 & & & \\
\hline & \multirow{3}{*}{ Total } & & $2.1 \%$ & $11.1 \%$ & $14.3 \%$ & $40.0 \%$ & $50.0 \%$ & & & \\
\hline & & & 48 & 36 & 7 & 5 & 4 & & & \\
\hline & & & $100.0 \%$ & $100.0 \%$ & $100.0 \%$ & $100.0 \%$ & $100.0 \%$ & & & \\
\hline
\end{tabular}

are prescriptions with poly pharmacy, geriatric patients with many comorbid conditions, multiple prescribers for a single patient, reduced patient compliance, improper conduct of medication history interview etc. From our clinical ward round experiences, we found a large no. of DDIs occurring that were adversely causing risks to the patients. There were situations where the patients had to suffer not only the burden of the cost of drugs but also the subsequent cost of drugs used for the treatment of the adverse reactions of the drugs as well as it resulted in reduced effectiveness of therapy, worsening of co-morbidities, signs and symptoms, drug toxicity etc. We strongly believe that clinical pharmacist interventions in such a scenario could bring a change to this by preventing the occurrence of potential DDIs that could cause harm to the patients. This influenced us in selecting our topic "A Prospective study on clinically relevant drug-drug interactions by systematic review of case files".

The study was of prospective interventional type which included a total of 200 inpatients randomly selected from departments of General Medicine, Gastroenterology, Pulmonology and Nephrology. Of which 100 patients were evaluated for DDIs in Phase I. An interventional phase was carried out before collecting data from another set of 100 randomly selected patients in phase III to check the effectiveness of the interventional phase. Out of the total 200 patients selected $110(n=200,55 \%)$ were males and $90(n=200,45 \%)$ females. Males experienced a total no. of 50 (58\%) DDIs in Phase I and 17 (39.6\%) of DDIs in Phase III. Females experienced a total no. of 36 (42\%) in Phase I and 19 (60.4\%) in Phase III with a $P$ value of 0.997 which indicated that gender had no influence on the drug-drug interactions. The patients were equally selected from all the 4 departments. Male patients were predominated over females in overall population and also in study population. This is similar to a study by Akram Ahmad et al. ${ }^{14}$ The average age of the study population was found to be $51.83 \pm 20.86$ years in our study whereas the avg. age was found to be $67.25 \pm 0.2$ years in a study conducted by Mandavi kashyap et al. ${ }^{15}$ This study revealed that as the no. of drugs/ prescription increased the no. of DDIs were also found to be increased. (Table 1) Since the obtained $P$ value was 0.00 , the relationship between no. of drugs prescribed and no. of DDIs/ prescription was statistically significant. The results were comparable to a study by $\mathrm{H}$. Sharifi et al. ${ }^{16}$ The total number of potential drug-drug interactions in the study was found to be 49 , out of which 368 (74.34\%) was found in Phase I and 127 (25.65\%) was found in Phase III. This indicated a significant decrease in the number of potential drug-drug interactions in phase III after the interventional phase (phase II). The result of the study was similar to the study by Ghulam Murtaza et al. ${ }^{12}$ The study done by Kumara Swamy RC et al. in Karnataka in 2014 revealed that as the no. of drug / prescription increased, DDIs also increased. ${ }^{8}$ Similarly our study revealed that as the no. of drugs / prescription increased, the no. of DDIs also increased. The result was statistically significant since the $P$ value was less than 0.005 .

The study done by Huda Kafeel et al. showed that, out of 202 interacting prescriptions $73(36.13 \%)$ had major interactions, 28 (13.79\%) had moderate interactions and $16(7.92 \%)$ had minor interactions, ${ }^{7}$ where on our study, out of 495 DDIs 157(31.71\%) had major interactions, (Figure 3) 315 (63.63\%) had moderate interactions and 23 (4.64\%) had minor interactions. Out of 368 DDIs in phase I and 127 DDIs in phase III, occurrence of major DDIs were $136(74.3 \%)$ and 21 (25.65\%) in phase I and phase III respectively, occurrence of moderate DDIs were 217 (43.83\%) and $98(19.79 \%)$ in phase I and phase III respectively and the occurrence of minor DDIs were $15(4 \%)$ and 8 (6.29\%) in phase I and phase III respectively. The Pearson Chi square test resulted in $P$ value $<0.05$ which implies that there is a statistically significant reduction in the number of major, moderate and minor drug-drug interactions in phase III after the interventional phase.

The interactions were classified into $\mathrm{C}, \mathrm{D}$ and $\mathrm{X}$ categories according to Lexicomp Risk Rate. C category interactions were found to be most common and X category interactions were found to be least (Figure 4). Out of 495 interactions, 274 (55.35\%) of interactions belonged to C category, 


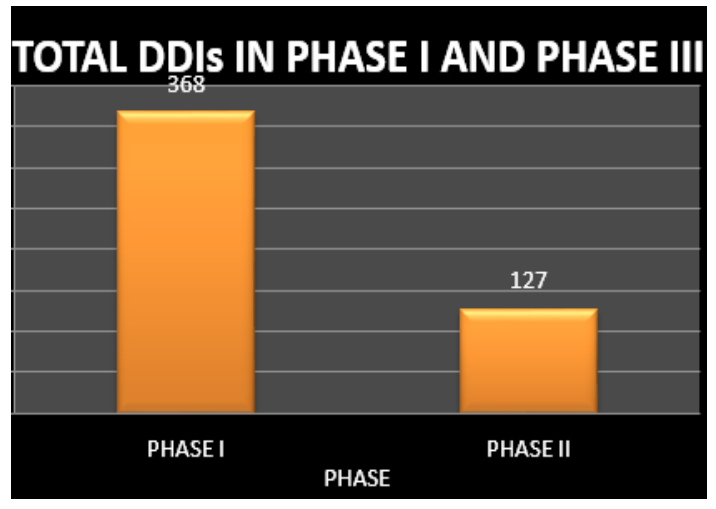

Figure: 1 Total number of DDIs in pre and post interventional phase.

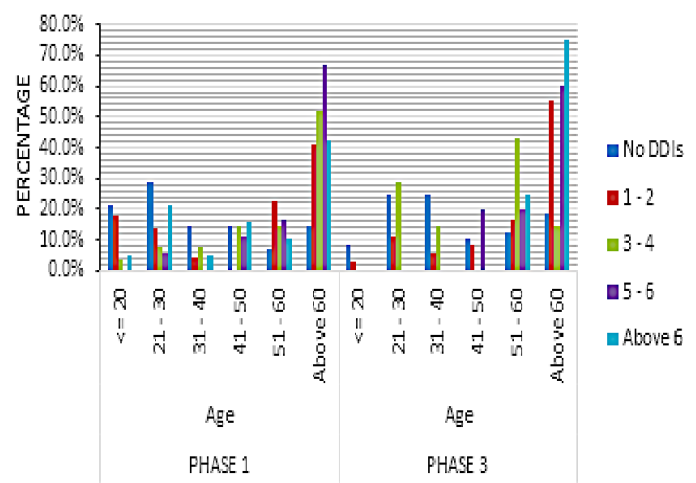

Figure 2: Relationship between age and DDIs in pre and post interventional phase.

$106(21.41 \%)$ of interactions belonged to D category and $66(13.33 \%)$ of interactions belonged to X category. Out of the total 274(55.34\%) DDIs with C risk rate, 193 (70.43\%) of it was found in Phase I and 81 (29.5\%) was found in Phase III. Out of the total 159 (32.12\%) DDIs with D Risk Rate, 89 (55.9\%) of it was found in Phase I and 70 (44\%) was found in Phase III. Out of the total 66 (13.33\%) DDIs with X Risk Rate, $55(83.33 \%)$ of it was found in Phase I and 11 (16.66\%) was found in Phase III. The Pearson Chi square test resulted in $P$ value $<0.05$ which implies that there is a statistically significant reduction in the number of $\mathrm{X}, \mathrm{D}$ and $\mathrm{C}$ drug-drug interactions in phase III after the interventional phase. The findings correlated with the findings of the study conducted by Gulcebi Idriz Oglu et al. ${ }^{17}$

During this study the effectiveness of clinical pharmacist intervention was assessed, regular monitoring and reporting reduces the drug interaction. Many of the potential drug-drug interactions can be avoided with monitoring the patient closely or with the use of alternative medications. Clinical pharmacists can play a very important role in identifying and monitoring potential drug-drug interactions and to select appropriate dosage or therapy adjustments.

The majority of DDIs were of pharmacokinetic type. Out of 495 DDIs, 228 (46\%) were pharmacokinetic DDIs, 154 (31.11\%) were pharmacodynamic DDIs and 115 (23.23\%) DDIs were of unknown type (Figure 6). This was in stark contrast to a study by Nithin Kothari et al. which showed that $55 \%$ of the DDIs were of pharmacodynamic type. ${ }^{18}$ Pharmacokinetic drug interactions deal with the modification of the effect of the drug caused by differences in the absorption, distribution, metabolism or excretion of the drugs.

\section{RISK RATE ACCORDING T0 LEXICO}

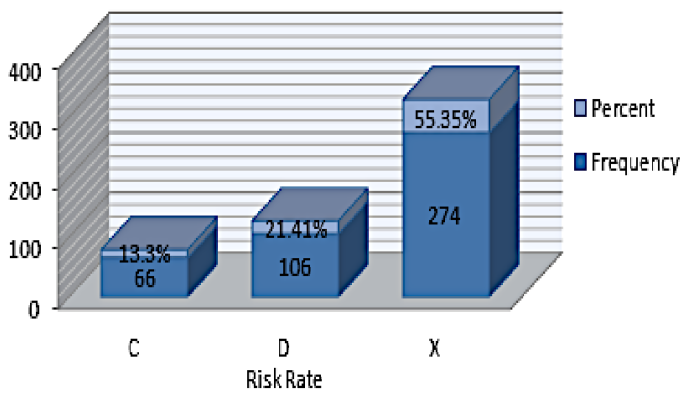

Figure 4: Risk rate percentage according to Lexicomp.

\section{Percentage of Major DDls}

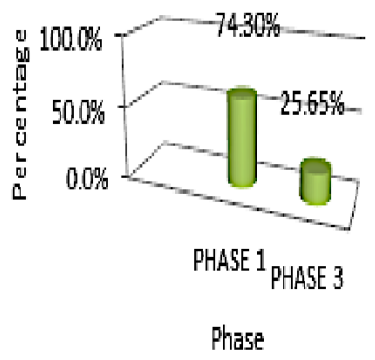

Percentage of Major DDls

Figure 3: Major DDIs in pre and post interventional phase.

The study revealed that out of the total 115 (23.23\%) Probable DDIs, 73 (63.47\%) were found in Phase I and 42 (36.5\%) in Phase III (Figure 5) with $P$ value $=0.03$ and Chi square $=9.046$. Out of the total $208(42 \%)$ Possible DDIs, 173 (83.17\%) were found in Phase I and 35 (16.82 \%) in Phase III with $P$ value $=0.00$ and Chi square $=61.540$. Out of the total 172 (34.74\%) Doubtful DDIs, 122 (71\%) were found in Phase I and 50 $(29 \%)$ in Phase III with $P$ value $=0.00$ and Chi square $=20.554$. Pearson Chi Square test resulted in $P$ value $<0.05$, which implies that there is a statistically significant reduction in the probable, possible and doubtful drug-drug interactions in phase III after the interventional phase. The results were comparable to study by Roheena Zafar et al. ${ }^{19}$ In this study a total of 167 changes in laboratory values were found in the study population of which 118 (70.65\%) was seen in Phase I and 49 (29.34\%) was seen in Phase III (Figure 7). This was comparable to study done by Jeannette E.F et al. where the changes in the laboratory values were found to be $59.9 \%$ in the study population. ${ }^{20}$ Out of 85 interventions, $69(81.17 \%)$ interventions were provided for the drug-drug interactions in phase I and $16(18.82 \%)$ interventions were provided in phase III (Figure 8$)$. This was comparable to the study done by Jeannette E.F. et al. where the total percentage of interventions was found to be $72 \%$ in the study population. ${ }^{3}$

\section{CONCLUSION}

The study revealed that 495 drug-drug interactions occurred in the study population, where majority of the interactions were of pharmacokinetic type. There was a drastic reduction in the occurrence of major DDIs after the interventional phase which showed a positive impact of pharmacist intervention on preventing the occurrence of drug-drug interactions that could otherwise cause harm to the patients leading to increased cost 


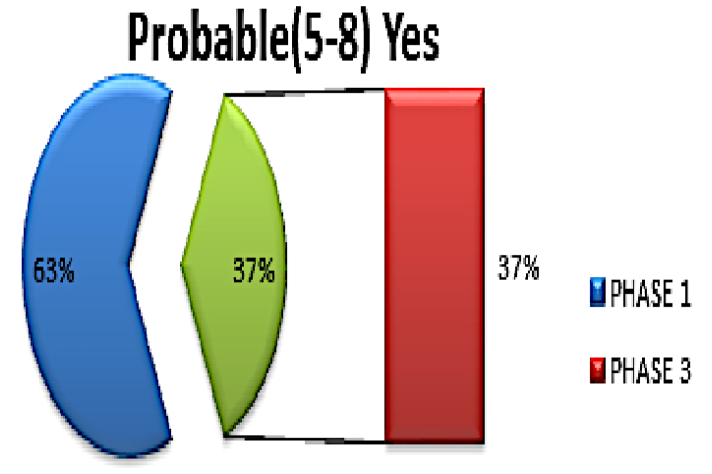

Figure 5:Proabale DDIs in pre and post interventional phase.

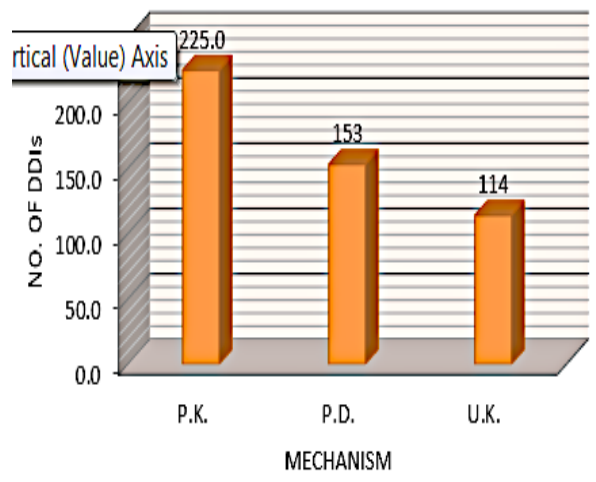

GDDls

Figure 6: DDIs based on mechanism of interactions [Pharmacokinetic (P.K.), Pharmacodynamic (P.D.), Unknown (U.K.)].

of burden, reduced effectiveness of therapy, worsening of co-morbidities etc. Given their causation, remedies and counseling, a clinical pharmacist plays an important role in the practice of safe medication and successful treatment.

\section{ACKNOWLEDGEMENT}

The authors thank Dr. Dilip C., H.O.D, Pharmacy practice for his firm guidance and also extend sincere gratitude to the physicians, Dr. Azharul Haque, Dr. Saju Xavier, Dr. Jaffer C.P., and Dr. Ganesh V.M. of Kims Alshifa Hospital for guiding us throughout the project giving us necessary support.

\section{CONFLICT OF INTEREST}

The authors declare no conflict of interest.

\section{ABBREVIATIONS}

DDI: Drug drug interaction; PK: Pharmacokinetic; PD: Pharmacodynamic; OTC: Over the counter; I.P: Inpatient.

\section{REFERENCE}

1. Ylva B, Kari L, Marine LA, Tuomas K, Bjorn M, Marie-Louise O, et al. Drug-drug interaction database designed for clinical decision support systems. Eur J Clin Pharmacol. 2009;65(6):627-33.

2. Aronson JK. Classifying drug interactions. Br J Clin Pharmacol. 2010;58(4):1549.

3. Preksha AB, Supriya DM, Varsha JP. Evaluation of potential drug-drug interactions in patients of Emergency Medicine Department at a tertiary care teaching hospital: A prospective study. Int J Sci Study. 2015;3(5):425-9.

\section{Change in Lab Values due to DDls}

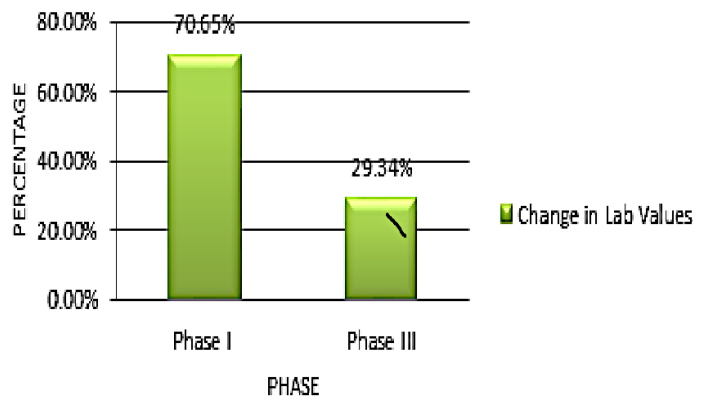

Figure 7: Changes in laboratory values observed during pre and post interventional phase.

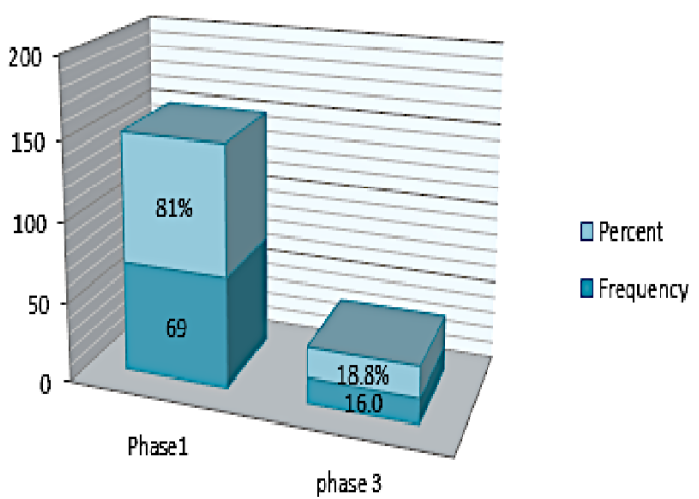

Figure 8: Interventions done for DDIs in pre and post interventional phase.

4. John P, Pamela L, Smith B. Common drug interaction leading to adverse drug events in ICU management and pharmacokinetic consideration. Australas Med J. 2011;4:625-57.

5. Kirsten KV, Hege SB, Tron AM, Aasmund R. Comparison of drug related problems in different patient groups. Br J Clin Pharmacol. 2007:63(2):187-95.

6. David WB, Shobha P, DerHeleen SV, Alisha DT, Amrita AD, Douglas SB. The impact of computerized physician order entry on drug-drug interactions prevention. JAMIA. 2012;20(3):489-93

7. Lucian LL, David WB, David JC, Nan L, Laura AP, Jonathan MT, et al. Effect of computerized physician order entry and a team intervention on prevention of serious medication errors. JAMA. 1998;280(15):1311-6.

8. Kumara SRC, Jignesh UR, Bushipaka R, Radadiya M, Sowmya B, Dhruvil P. Prevalence of Polypharmacy And Drug to Drug Interactions in a Tertiary Care Teaching Hospital. J Drug Health Patient Saf. 2014;5(10):2230-8407.

9. Zwart-van R, Esther VU, Ten BMJ, Wouter WSV, Antoine CGE. Frequency and nature of drug-drug interactions in a dutch university hospital. Br J Clin Pharmacol. 2009;68(2):187-93.

10. Huda K, Ramsha R, Hina Q, Jaweria B, Mehreen J, Rabia S, et al. Possibility of drug-drug interaction in prescription dispensed by community and hospital pharmacy. J Pharmacol Pharm. 2014;5(4):401.

11. Semih OS, Ahmet S. Food and drug interactions: A general review. Acta Sci Pol Technol Aliment. 2014;13(1):440-9.

12. Ghulam M, Muhammed YGK, Saira A, Shujaat AK, Tahir MK. Survillance of the potential drug-drug interactions in the medicine department of a tertiary care hospital. J Clin Diagn Res. 2012;6(7):1258-61.

13. Daniel CM, Edward PA, Jacob A, Amy JG, Philip DH, Robin CVB, et al. Identification of serious drug-drug interactions: Results of the partnership to prevent drug-drug interactions. J Am Pharm Assoc. 2004;44(2):142-51.

14. Akram A, Mehul R, Bharti C. Evaluation of potential drug-drug interactions in general medicine ward of teaching hospital in Southern India. J Clin Diagn Res. 2015;9(2):10-3.

15. Mandavi K, Sanjay DC, Atul S, PramilT. Study on drug-drug interactions and their predictors. Saudi Pharm J. 2013;11(4):191-5.

16. Ralph El, Jeffrey KA. Drug-drug interactions-a preventable patient safety issue? The Lancet. 2000;356(9237):1255-9. 
17. Gulcebi IO, Wen CP, Rossert J. Potential drug-drug interactions in a medical intensive care unit of a university hospital. J Pharmacol Pharm. 2016;46(3):812-9.

18. Nitin K, Barna G. Study on Potential drug-drug interactions among medications prescribed to hypertensive patients. J Clin Diagn Res JCDR. 2014;8(11):625-9.

19. Roheena Z, Kamran AC, Muhammed Z. Study on potential drug-drug interac- tions in the neurology ward of a tertiary care hospital in Peshwar, Pakistan. Tr J Pharm Res. 2017;16(10):2521-6.

20. Zwart-van JEFR, Esther VU, Maarten JTB, Wouter WVS, Antoine CE. Frequency and nature of drug drug interactions in Dutch university hospital. BJ of Clin Pharmacol. 2009;68(2):187-93.

Article History: Submission Date : 05-12-2018; Revised Date : 17-02-2019; Acceptance Date : 29-03-2019.

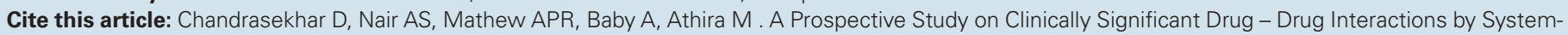
atic Review of Case files in a South Indian Tertiary Care Hospital . J Young Pharm. 2019;11(3):285-90. 